\title{
Thermal activation analysis of the structural and phase transformations in the $\mathrm{Zr}-\mathrm{Cu}-\mathrm{Nb}$ amorphous alloy
}

\author{
N.A. Minina ${ }^{1}$, V.A. Yermishkin ${ }^{1}$, Yu.D. Lepeshkin ${ }^{1}$ and N.L. Fedotova ${ }^{2}$ \\ ${ }^{1}$ Baikov Institute of Metallurgy and Materials Science, RAS, Leninskii pr. 49, Moscow, Russia \\ ${ }^{2}$ Bardin Central Scientific Research Institute of Ferrous Metallurgy, Vtoraya Baumanskaya ul. 6, Moscow, Russia
}

\begin{abstract}
In the present work, the procedure of the estimation of the thermal activation parameters from the data of dilatometric measurements and the results of its application to the $\mathrm{Zr}-\mathrm{Cu}-\mathrm{Nb}$ amorphous alloy discussed. The determination of the thermal activation parameters of the processes occurring in materials under known temperature-force conditions can be useful for the identification of the structural mechanisms of phase transformations and the evolution of defect structure. We used the data of dilatometric measurements for evaluating the effective activation energy. This method exhibits some advantages over the conventional one due to the design features of dilatometers. First, it ensures the precise measurement of strains; second, the assigned temperature regime is very precisely maintained both at the stage of heating and upon isothermal holding; third, it ensures a high-speed continuous record of the experimental data. The developed method of evaluating the effective activation energy from the results of dilatometric experiments provides statistically reliable results. The data of the photometric analysis of structure images are in accordance with the results of dilatometric experiments.
\end{abstract}

\section{Introduction}

The determination of the thermal activation parameters of the processes occurring in materials under known temperature-force conditions can be useful for the identification of the structural mechanisms of phase transformations and the evolution of defect structure. Furthermore, the thermal activation parameters control the kinetics of these processes and, thus, form the required level of the physical characteristics of materials. The existing methods of thermal activation analysis (TAA) are based on the treatment of the experimental temperature-rate dependences of the structure-sensitive characteristics of materials by the equation recorded by analogy with Arrhenius' formula for the chemical reaction rate [1]:

$$
\dot{\varepsilon}_{=} \dot{\varepsilon}_{\sigma} \cdot \exp [-U(\sigma) / k T]
$$

where: $z$ is the plastic strain rate, $U(\sigma)$ is the activation energy for the process, $\varepsilon_{0}$ is the constant of the process, $k$ is the Boltzmann constant, $T$ is the temperature in the absolute degrees. The preexponential factor $\varepsilon_{0}$ is usually represented as the product: $\varepsilon_{0}$ is $=b \rho l$, where: $b$ is the Burgers vector of dislocations, $\rho$ is their density, and $l$ is the mean free path of dislocation after overcoming the energy barrier which separates its two stable positions. In the analysis, $\varepsilon_{0}$ is assumed to be independent of temperature and the stress in a sufficiently wide interval, but this assumption, as a rule, is not fulfilled. The conventional TAA methods give the best results in the case where they use the data of the experiments on creep, for which the conditions $T=$ const and $\sigma=$ const are fulfilled, and the data obtained upon jumpwise changes in temperature and stress [2], but this case requires a large volume of experiments. We used the data of dilatometric measurements for evaluating the effective activation energy. This method exhibits some advantages over the conventional one due to the design features of dilatometers. First, it ensures the precise measurement of strains; second, the assigned temperature regime is very precisely maintained both at the stage of heating and upon isothermal holding; third, it ensures a high-speed continuous record of the experimental data. In particular, at a heating rate of $10^{\circ} \mathrm{C} / \mathrm{min}$, the step of heating does not exceed $0.5^{\circ} \mathrm{C}$ per $1 \mathrm{sec}$, which makes it possible to obtain 20 temperature measurements for $10 \mathrm{~min}$ of strain. Earlier, it was established that the deformation kinetics upon isothermal holding of the sample in the dilatometer is well described by following the power dependence [3]: 


$$
\dot{\varepsilon}(t)=\varepsilon_{v} \cdot\left(1+t \cdot \exp \left[-\frac{v \sigma r}{k T}\right]\right)^{2}
$$

In Eq. (2), $\varepsilon_{0}$ means the strain of the sample at the beginning of isothermal annealing, $t$ is the chronometric time of the process, $n$ is the constant of the process, and

$k$ is the Boltzmann constant. The second member of the expression in the parentheses is the analog of the effective process time, which for the first time was introduced by Dorn [4] for the description of the universal curve of high-temperature creep of aluminum with the difference that the exponent in [4] contained the activation energy for self-diffusion $Q$ instead of $\mathrm{U}(\sigma)$.

\section{Method of the analysis of dilatometric data}

Let us represent the heating process as a sequence of isothermal processes at temperatures elevating with a constant step $\Delta \mathrm{T}$ and assume that the preexponential factor $\varepsilon_{0}$ for four sequential measurements is changed insignificantly. Then we obtain Eq. (3), which implicitly includes the only indeterminate $\mathrm{U}(\sigma)[5]$ :

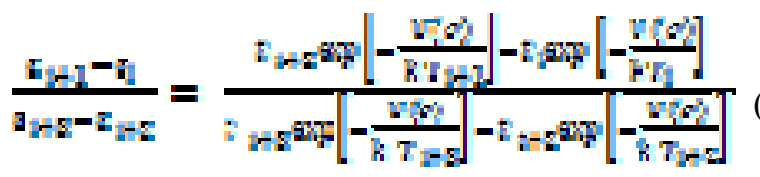

where: $i, i+1, i+2, i+3$ are the indices of four sequential dilatometric measurements. This equation can be solved by numerical method with the substitution of the corresponding dilatometric variables, the initial $\mathrm{U}(\sigma)$ value, the step of its change, and the assigned precision of calculations. The results of this work were obtained with the aid of a computer program designed for the calculations. The calculated data were interpreted by using distribution functions. Each $\mathrm{U}(\sigma)$ value was correlated to the fraction with the numerator containing the number of solutions, at which Eq. (2) becomes an identical equation at the assigned precision of calculations $(0.01 \mathrm{eV}),\left(\mathrm{n}_{\mathrm{j}}\right)$, and the denominator representing the total number of the data used for the calculation $\left(\mathrm{N}_{\mathrm{j}}\right)$. These fractions were subjected to renormalization with the aid of Eq. (4) derived from the proportion: to impart the sense of the probability $\left(p_{j}\right)$ of the appropriate activation energy value to the obtained result.

$$
p_{j}=\frac{\pi_{j}}{N \cdot \Sigma^{m}-\frac{r_{1}}{N}}
$$

where $\mathrm{j}$ is the index of the sequence of the assigned $\mathrm{U}(\sigma)$ values, and $m$ is the number of the members of this sequence. The analysis of the data was performed in individual stages: upon heating, upon isothermal holding, and upon cooling. In this case, the individual sections with the monotonic behavior of the curve were selected, depending on the character of the change in deformation as a function of temperature for each stage. The average effective activation energies were evaluated from Eq. (5):

$$
\tilde{\mathrm{U}}=\sum_{\mathrm{i}=1}^{\mathrm{m}} \mathrm{U}(\sigma)_{\mathrm{i}} \cdot \mathrm{p}_{\mathrm{p}}
$$

\section{Results and discussion}

The experimental part of the work was performed with a thin ribbon $10 \mathrm{~mm}$ wide and $0.066 \mathrm{~mm}$ thick from the $\mathrm{Zr}-\mathrm{Cu}-\mathrm{Nb}$ alloy in the nanostructural state. The dilatometric micro samples were examined with a DL1500-RH high-speed dilatometer at a heating rate of 0.5 $\mathrm{K} / \mathrm{sec}$. Figure 1 shows the dilatometric curve of the alloy for the entire thermal cycle of the experiment. It is evident that the thermal deformations of the sample reflect not only its thermal expansion, but also the development of phase transformations and structural rearrangement in it.

The above procedure of thermal activation analysis was used to calculate the functions of the activation energy distribution in all monotonic behavior sections of dilatometric curve at the stages of heating, cooling, and isothermal annealing. The temperature intervals of these sections and the average values of the effective activation energies calculated from the functions of the effective activation energy distribution are given in Table 1.

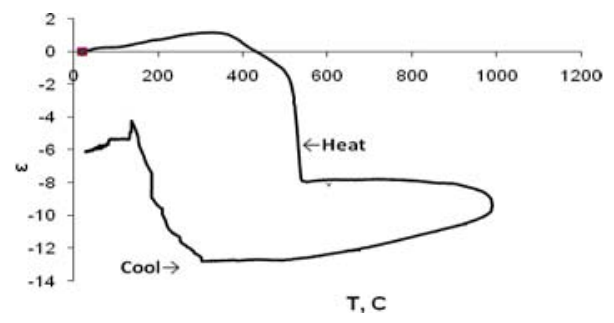

Fig. 1. Dilatometric curve of the $\mathrm{Zr}-\mathrm{Cu}-\mathrm{Nb}$ based nanostructural alloy.

Figure 2 shows the distribution functions of the effective activation energies for sections 1, 2, 3 .

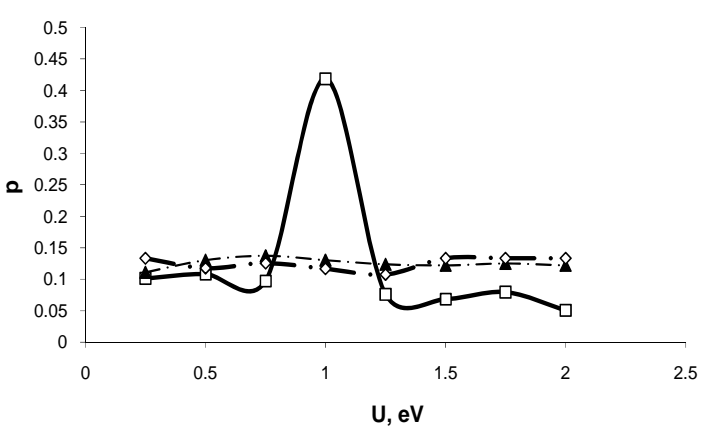

Fig. 2. Distribution functions of the effective activation energies for sections $1,2,3$. ( $\square-1, \mathbf{\Delta}-2, \diamond-3)$ 
Table 1. Temperature intervals of the monotonic behavior of dilatometric curve and the corresponding average values of the effective activation energies

\begin{tabular}{|l|l|l|l|}
\hline No. & \multicolumn{2}{|l|}{ Boundaries of the temperature intervals } & $\mathrm{U}(\sigma), \mathrm{eV}$ \\
\hline & $\mathrm{T}_{\text {begin }}, \mathrm{K}$ & $\mathrm{T}_{\text {end }}, \mathrm{K}$ & \\
\hline 1 & 294.6 & 643.4 & 1.01 \\
\hline 2 & 644.3 & 808.5 & 1.12 \\
\hline 3 & 800.5 & 818.13 & 1.14 \\
\hline 4 & 834.13 & 1261.3 & 1.08 \\
\hline 5 & 1261.4 & 581.6 & 1.15 \\
\hline 6 & 581.4 & 418.84 & 1.10 \\
\hline 7 & 418.09 & 294.5 & 1.14 \\
\hline
\end{tabular}

The same distribution functions for sections $4,5,6,7$ of the dilatometric curves are shown in Fig. 3.

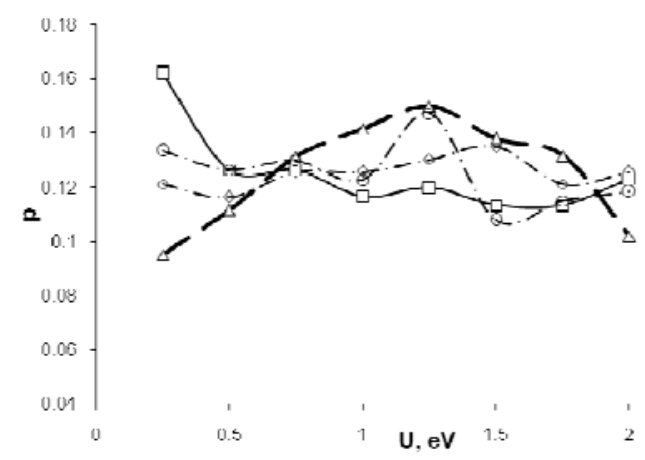

Fig. 3. Distribution functions $U(\sigma)$ for sections $4,5,6,7$ ( $\square-4$, $\Delta-5, \circ-6,-\diamond-7)$

It is seen from Figures 2 and 3 that the distribution functions $U(\sigma)$ for different sections can strongly differ in form because of the difference in temperature conditions. Nevertheless, for the alloy under investigation, the values of the effective activation energies for the thermally stimulated strains little differ in value (Table 1) and are close to the activation energy for the vacancy migration in the zirconium base alloys

[6]. This well agrees with the fact that the nanomaterials obtained by ultra-rapid solidification are usually supersaturated by vacancies. At the same time, the character of dilatometric curve shows substantial changes occurring in the phase composition and defect structure upon heating and cooling. Figure 4 represents the graph of the change in the difference in the deformations upon heating and cooling as a function of temperature. It is seen from the figure that the phase

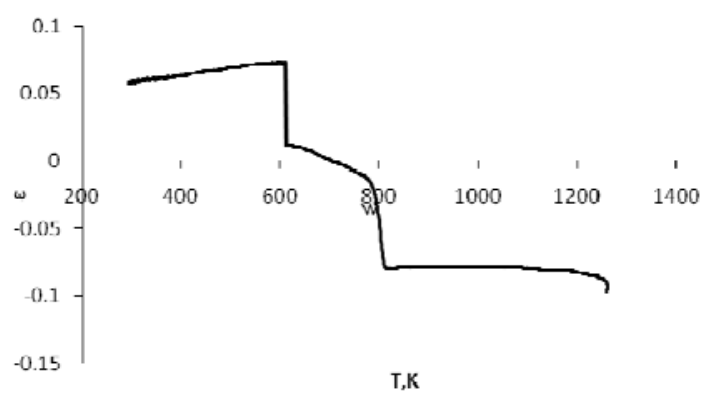

Fig.4. Difference in the strains upon heating and cooling as a function of temperature. transformations of martensitic type characterized by a high rate of change in the specific volumes of the forming phases occur at temperatures of $607 \mathrm{~K}$ and $800 \mathrm{~K}$. The temperature interval between these temperatures is characterized by well-developed structural transformations, which, apparently, occur by the mechanism of the nucleation and growth of structure elements. These conclusions agree with the data obtained by the measurement of the structural damage of the sample surface by the method of the photometric analysis of structure images (PHASI) [7] shown in Figure 5. The $\mathrm{Y}$-axis represents the alloy structural damage $\mathrm{P}(\mathrm{t})$ determined by the formula:

$$
P(t)=\frac{a_{t}\left(N-a_{0}(N)\right.}{a_{0}(N)}
$$

where: $a_{t}(N)$ is the average spectral density of the brightness of the visible light reflection from the sample surface in the interval of number $\mathrm{N}$ after the temperature action on the sample, and $\mathrm{a}_{0}(\mathrm{~N})$ is the same, but before heating of the sample. Figure 5 shows two curves corresponding to the brightness range of reflection with the increased absorption ability $(\Delta)$ and brightness of the visible light reflection from the sample to the interval surrounding the maximum of the reflection brightness spectrum $(\square)$. These curves show the dependence of structural changes on the temperature of isothermal annealing for $10 \mathrm{~min}$. It is seen that the character of the reflection brightness abruptly changes at temperatures ranging from $600 \mathrm{~K}$ to $800 \mathrm{~K}$. Such behavior indicates the change in the concentration of the valence electrons, which interact with the quanta of visible light upon its reflection.

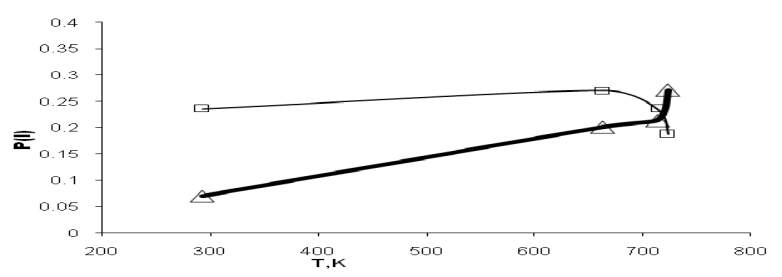

Fig.5. Structural damage of the alloy as a function of annealing temperature: $(\square)$ the curve is constructed according to the data on the change in the average spectral density of the reflection brightness in the interval surrounding the spectrum maximum and $(\Delta)$ the same, but in the spectrum interval with the increased absorption ability). 


\section{EPJ Web of Conferences}

\section{Conclusions:}

1. The developed method of evaluating the effective activation energy from the results of dilatometric experiments provides statistically reliable results.

2. The activation energies in different sections of the monotonic change in the dilatometric curve at all stages of the thermal cycle are close in value and correspond to vacancy migration energy.

3. The curve of the difference in strains formed at the stage of heating and cooling allows the establishment of temperatures of the phase transformations occurring by the martensitic mechanism; the field of the structural changes occurring by the mechanism of nucleation and growth lies between these temperatures.

4. The data of the photometric analysis of structure images are in accordance with conclusion 3. The work is supported by RFBR (RU) 07-0800381a; 09-08-00563a.

\section{References}

1. Evans A. G., Rawlings R. D., The thermally activated deformation of crystalline materials, Physica Status Solidi (B), 34, issue 1, pp. 9-31. (1973)
2. Minina N.A., Yermishkin V.A., Lepeshkin Yu.D., Fedotov N.L. Kinetics of the structural evolution of metals and alloys under temperature action. Fizika i Khimiya Obrabotki Materialov. No. 3, pp. 72-76 (2009)

3. Dorn J.E. The energy spectrum of the activation of creep./in Creep and recovery. Moscow. Metallurgy. pp. 291-325 (1961)

4. Yermishkin V.A., Minina N.A., Tomenko A.K. Thermal activation analysis of the dilatometric experiments of $\mathrm{Al}-15 \% . \mathrm{Zn}$ alloy. Materials of the XVI International Scientific-Technical Conference "Machine Building and Technosphere of the XXI Century", 14-19 September 2009, Sevastopol', pp. 225-230 (2009)

5. Orlov A.N., Trushin Yu.V. Energy of point defects in the metals. Moscow: Energiya-Atomizdat. P. 81 (1983)

6. Yermishkin V.A., Minina N.A., Fedotov N.L. Patent No. 2387978 "Method of photometric diagnostics of phase transformations in solids from the data of the brightness spectra analysis of the light reflection from their surface" publ. 27.04.2010, Byull. Izobr. No. 12, pp.1-11 (2010)

7. Yermishkin V.A., Lepeshkin Yu.D., Murat D.P., Ovchinnikov I.N. Method of photometric diagnostics of the structural state of materials according to the data of the analysis of the digital coded image of their surface. Patent No.2387974, Byull. Izobr. No. 13, P. 14. (2010) 